\title{
Transition to Stochastic Synchronization in Spatially Extended Systems
}

\author{
Lucia Baroni and Roberto Livi* \\ Dipartimento di Fisica, Universitá di Firenze, L.go E. Fermi, 5 - I-50125 Firenze, Italy \\ Istituto Nazionale di Fisica della Materia, UdR Firenze, L.go E. Fermi, 3 - I-50125 Firenze, Italy \\ Alessandro Torcini ${ }^{\dagger}$ \\ Dipartimento di Fisica, Universitá "La Sapienza", P.le A. Moro, 3 - I-00185 Roma, Italy \\ Istituto Nazionale di Fisica della Materia, UdR Firenze, L.go E. Fermi, 3 - I-50125 Firenze, Italy
}

(Draft version, November 4, 2018)

\begin{abstract}
Spatially extended dynamical systems, namely coupled map lattices, driven by additive spatio-temporal noise are shown to exhibit stochastic synchronization. In analogy with low-dymensional systems, synchronization can be achieved only if the maximum Lyapunov exponent becomes negative for sufficiently large noise amplitude. Moreover, noise can suppress also the non-linear mechanism of information propagation, that may be present in the spatially extended system. A first example of phase transition is observed when both the linear and the non-linear mechanisms of information production disappear at the same critical value of the noise amplitude. The corresponding critical properties can be hardly identified numerically, but some general argument suggests that they could be ascribed to the Kardar-Parisi-Zhang universality
\end{abstract}

\footnotetext{
*Electronic Address: livi@fi.infn.it URL : http://docs.de.unifi.it/ docs

${ }^{\dagger}$ Electronic Address: torcini@ino.it URL : http://www.ino.it/ ttorcini
} 
class. Conversely, when the non-linear mechanism prevails on the linear one, another type of phase transition to stochastic synchronization occurs. This one is shown to belong to the universality class of directed percolation.

PACS numbers: 05.45.-a,05.45.Jn,05.45.Ra,05.45.Xt,05.40.Ca

Typeset using REVTEX 


\section{Introduction}

The synchronization of dynamically coupled chaotic systems is a new interesting phenomenon that has attracted many studies and efforts in the last years. The first models and examples discussed in the literature concerned the case where the dynamical coupling is performed by a deterministic chaotic signal (either external or self-generated) acting on different trajectories of the same low-dimensional chaotic system (e.g., see [1]). Under quite general conditions, e.g. large enough coupling strength and sufficiently long evolution time, the two trajectories approach each other and, despite their chaotic nature, no more diverge. Beyond the intrinsic conceptual interest of "chaotic synchronization", the main motivations of these studies came from the possibility of identifying efficient mechanisms for controlling or suppressing chaos in deterministic signals, with promising perspectives of applications to the automatic control of devices and to cryptography.

On the other hand, applying a control mechanism to a deterministic system by a chaotic signal looks artificial with respect to the more realistic scenario, where the coupling could be produced by noise. It is worth stressing that deterministic chaos cannot be assimilated to a random process, despite in some cases they may yield equivalent results 1 . Accordingly, it is quite natural to raise the question if synchronization can be observed also when the chaotic coupling is substituted with a stochastic signal. The problem of "stochastic synchronization" has been investigated by several authors [2 6] considering two different initial conditions of the same low-dimensional chaotic system (e.g. the logistic map or the Lorenz system) and making them evolve by adding to both of them the same realization of noise. For sufficiently large values of the noise amplitude, the stationary probability measure associated with the chaotic dynamics may be modified by the addition of noise favouring the stabilization onto the same stochastic orbit. As argued by Pikovsky [2], this effect can be explained and quantitatively predicted by observing that, at some value of the noise amplitude, the Lyapunov exponent associated with the dynamics changes from positive to negative. A necessary condition for the occurrence of synchronization is that the dynamical system has expanding and contracting regions, so that noise may amplify the role of the latter against the former. For instance, synchronization cannot emerge from uniformly hyperbolic maps, like the Bernoulli shift. In Section I we discuss "stochastic synchronization" in simple maps, that we introduce as examples of different classes of models. The new question that we want to address in this paper is the following: under which conditions stochastic synchronization may occur in spatially extended dynamical systems coupled by the same realization of spatiotemporal noise? The models, that we shall study numerically, are the diffusively coupled map lattice (CML) [7] versions of those introduced in Section I. A comparison with the analysis therein worked out on stochastic synchronization in low-dimensional systems shows that the spatial structure makes this phenomenon even richer. Specifically, let us list hereafter the main results contained in this paper:

- Pikovsky's criterion [2] can be generalized to spatially extended systems : Stochastic synchronization occurs only if the maximum Lyapunov exponent $\Lambda$ [8] is negative. In

\footnotetext{
${ }^{1}$ For instance, the Monte Carlo procedure applied to an ergodic system yields the same equilibrium properties emerging from the Liouville measure associated with the dynamics.
} 
fact, we find that for noise-coupled CML models $\Lambda$ becomes negative for values of the noise amplitude $\sigma$ larger than a threshold value $\sigma_{\Lambda}$.

- It is well known that in spatially extended systems information may propagate with a finite velocity $V$ [9], due to spatio-temporal instabilities. For noise-coupled CML models, with smooth nonlinearities, the only relevant instability mechanism is the linear one, so that $V$ vanishes with $\Lambda$. This identifies a synchronization transition (PT1) at a threshold value $\sigma_{V} \equiv \sigma_{\Lambda}$, whose critical properties seem to belong to the Kardar-Parisi-Zhang (KPZ) universality class of interface roughening transition [10].

- We discuss also other models where deterministic dynamics or spatiotemporal noise may induce strong non-linear effects. In these cases we find that $V$ vanishes at a value of the noise amplitude $\sigma_{V}>\sigma_{\Lambda}$. A different kind of synchronization transition (PT2) is observed at $\sigma_{V}$ and its critical properties are found to belong to the universality class of directed percolation (DP) [11,12].

- In the latter case we find also that the time needed to achieve a synchronized state grows exponentially with the system size $L$ for $\sigma_{V}>\sigma>\sigma_{\Lambda}$ : this dynamical regime is analogous to the "stable-chaotic" phase observed in [13]. Conversely, this time grows logarithmically with $L$ for $\sigma>\sigma_{V}$ (see also [14]).

In Section II we introduce the models and the suitable indicators for describing the synchronization transitions induced by spatio-temporal noise in CMLs. The dynamical features of the different scenarios are analyzed in Section III. In Section IV, we study the critical properties associated with the transition to stochastic synchronization in spatially extended systems : the numerical analysis indicates that this phenomenon is a genuine nonequilibrium process, characterized by the scaling laws of KPZ and DP universality classes for PT1 and PT2, respectively. We expect that a suitable coarse-graining or renormalization approach could provide a rigourous ground for these results if one could map the noise-coupled CML dynamics onto the stochastic PDE's that identify the above mentioned universality classes. Unfortunately, we have not been able to work out any consistent derivation. Nonetheless, numerics allows one for drawing some conclusions about the problem at hand: they are summarized in Section V, together with some perspectives for future work on this subject.

\section{A SURVEY ON STOCHASTIC SYNCHRONIZATION IN LOW-DIMENSIONAL SYSTEMS}

Before entering the main topic of this paper, i.e. stochastic synchronization in spatially extended systems, it is worth presenting concepts, tools and properties characterizing the same kind of phenomenon in low-dimensional dynamical systems. In this section we consider some simple models, that exemplify the general features of this phenomenon.

The basic model equation is the stochastic map

$$
x^{t+1}=f\left(x^{t}\right)+\sigma \cdot \eta^{t}
$$

where the state variable $x^{t}$ is a real quantity depending on the discrete time index $t, \sigma$ is the amplitude of the time dependent random variable $\eta^{t}$, uniformly distributed in the interval [$1,1]$, and $f(x)$ is a map from $\mathcal{S} \subseteq \mathbb{R}$ into the interval $\mathcal{I} \subseteq \mathbb{R}$. Stochastic synchronization can 
be investigated by considering two different initial conditions, $x^{0}$ and $y^{0}$ of dynamics (1) with the same realization of additive noise $\eta^{t}$. More precisely, we assume that the corresponding trajectories, $x^{t}$ and $y^{t}$, generated by (8) may synchronize if their distance

$$
d(t)=\left|x^{t}-y^{t}\right|
$$

becomes smaller than a given threshold $\Delta$, usually assumed much smaller than unit. This definition allows one to identify two natural quantities associated with the synchronization of trajectories: the first passage time $\tau_{1}(\Delta)$, i.e. the first instant of time at which $d(t) \leq \Delta$ and the synchronization time $\tau_{2}(\Delta)$, i.e. the interval of time during which $d(t)$ remains smaller than $\Delta[15]$. We want to point out that, in general, both $\tau_{1}(\Delta)$ and $\tau_{2}(\Delta)$ depend on the initial conditions and on the realization of noise. Accordingly, their averages over both ensembles have to be considered as the quantity of interest for our analysis. For the sake of simplicity we shall indicate these averaged times with the same notations $\tau_{1}(\Delta)$ and $\tau_{2}(\Delta)$.

In all the numerical simulations, for sufficiently small value of $\Delta$ (typically $\Delta<10^{-7}$ ), the results do not depend on the choice of $\Delta$. Moreover, one can assume that the trajectories have eventually reached the synchronized state if, after $\tau_{1}(\Delta), d(t)$ remains smaller than $\Delta$ for arbitrarily large integration times. This amounts to say that $\tau_{2}(\Delta)$ goes to infinity with the integration time. This heuristic definition of the synchronized state can be replaced by a more quantitative criterion: according to Pikovsky [2], for a given value of $\sigma$ the synchronized state occurs if the Lyapunov exponent $\Lambda$ of dynamics (11) is negative. This indicator is defined as follows:

$$
\Lambda=\lim _{t \rightarrow \infty} \frac{1}{t} \ln \prod_{j=1}^{t}\left|\frac{\xi^{j}}{\xi^{j-1}}\right|
$$

where the dynamical variable $\xi^{t}$ obeys the "linearized" dynamics

$$
\xi^{t+1}=\frac{\partial x^{t+1}}{\partial x^{t}} \xi^{t}
$$

and the derivative is computed along the trajectory given by (1). We want to remark that $\Lambda$ is well defined for deterministic dynamics (e.g. the $\sigma=0$ case in (11) ). In the framework of the linear stability analysis a positive (negative) $\Lambda$ measures the average exponential expansion (contraction) rate of nearby trajectories. For $\sigma \neq 0$ we are faced with stochastic trajectories and it is not a priori obvious if $\Lambda$ is still a meaningful quantity [16]. On the other hand, eq. (4) does not depend explicitely on $\eta^{t}$ and it is formally equivalent for both the noisy and the noise-free cases. However, the presence of noise modifies the evolution of the system w.r.t the noise-free case and, accordingly, also the tangent space dynamics. We have verified numerically that $\Lambda$ is a self-averaging asymptotic quantity also for dynamics (1) with $\sigma \neq 0$. It can be interpreted as the average exponential expansion (contraction) rate of infinitesimal perturbations of stochastic trajectories generated by the evolution rule (1) . In particular, its value is found to depend on $\sigma$, but not on the realization of noise.

Let us remark that it is quite simple to argue why $\Lambda<0$ implies $\tau_{2} \rightarrow \infty$ : After some finite time $\tau_{1}(\Delta), d(t)$ has decreased below a small threshold $\Delta$ and the trajectories can be viewed as a perturbation of each other. Linear stability implies that their distance will keep 
on decreasing exponentially with an average rate $\Lambda$, so that, within numerical precision, they will converge rapidly onto the same trajectory.

As a first example, we consider the continuous map shown in Fig. 1:

$$
f(x)= \begin{cases}-c \tanh (b(1+x)) & \text { if } x<-1 \\ a x(1-|x|) & \text { if }|x|<1 \\ c \tanh (b(1-x)) & \text { if } x>1\end{cases}
$$

where $\mathcal{S} \equiv \mathrm{R}$ and $\mathcal{I}=[-1,1]$. We choose the parameter values $a=4$, and $c=0.5$, so that (5) can be viewed as a sort of anti-symmetrized version of the logistic map at the Ulam point, taking values over the whole real axis. It can be easily shown that for $\sigma=0$ and independently of $b, \Lambda=\ln 2$, i.e. map (5) is chaotic. Notice that the noise term of amplitude $\sigma$ extends $\mathcal{I}$ to the interval $[-1-\sigma, 1+\sigma]$. We have verified numerically that, for any value of $b$ and for $\sigma$ larger than a threshold value $\sigma_{\Lambda}, \Lambda$ becomes negative and after some finite time $\tau_{1}$ a synchronized state is eventually achieved. In particular, we find that $\sigma_{\Lambda}$ is strongly dependent on $b$ : for instance, we have obtained $\sigma_{\Lambda}=1.2$ for $b=2$ and $\sigma_{\Lambda}=0.019$ for $b=1000$. As recently found also by Lai and Zhou [6] for a similar mapping, this result indicates that symmetric, i.e. zero-average, noise can yield stochastic synchronization.

It is worth mentioning that some time ago Herzel and Freund [5] conjectured that stochastic synchronization can be achieved only if noise has a non-zero average. They were led to such a conclusion by studying stochastic synchronization for the case of a map $f$ of the unit interval into itself, i.e. $\mathcal{S} \equiv \mathcal{I}=[0,1]$. In such a case the application of the stochastic evolution rule (四) demands the adoption of some further recipe for maintaining the state variable $x^{t}$ inside the unit interval, when $\sigma \neq 0$. For instance, one can choose the following reinjection rule $x^{t+1} \rightarrow x^{t+1}+1\left(x^{t+1} \rightarrow x^{t+1}-1\right)$ if $x^{t+1}<0\left(x^{t+1}>1\right)$. As discussed in [5], any recipe of this kind yields an effective state-dependent noise, that does not preserve the original symmetry of the stochastic process $\eta^{t}$, thus acquiring a non-zero average value. The above described example and the results obtained in [6] disprove their conjecture.

Nonetheless, an interesting observation is contained in [5]: the stochastic evolution rule (1) for maps definite on a finite interval induces strong non-linear effects due to the discontinuities introduced into the dynamics by the state-dependent noise. This strong non-linear character of the dynamics is irrelevant for stochastic synchronization in low-dimensional systems; conversely, it reveals a crucial property for discriminating between different critical behaviours in high-dimensional systems, as we shall discuss in Section IV.

It is also worth considering that even the presence of non-zero average noise does not necessarily guarantee stochastic synchronization. For instance, a counterexample is provided by considering dynamics (11) for the logistic map at the Ulam point:

$$
f(x)=4 x(1-x)
$$

whose noise-free dynamics is mixing. The state-dependent noise modifies the probability measure of $x^{t}$ in such a way to increase the weight of the contracting regions of the map. On the other hand, $\Lambda$ remains positive as well as $\tau_{2}$ remains finite for any value of $\sigma \in$ $[0,1]$, despite they can be made so small and so large, respectively, to produce an apparent synchronization effect. As discussed in [4, 5], misleading results can be obtained in this case due to the finite computational precision of numerical simulations.

As a final example, we consider the map 


$$
f(x)= \begin{cases}b x & \text { if } 0<x<1 / b \\ a+c(x-1 / b) & \text { if } 1 / b<x<1\end{cases}
$$

Its dynamics converges to a stable periodic attractor: for $b=2.7, a=0.07$ and $c=0.1$ this is a period-3 orbit with negative Lyapunov exponent, $\Lambda=\ln \left(c b^{2}\right) \approx-0.316$. Numerical

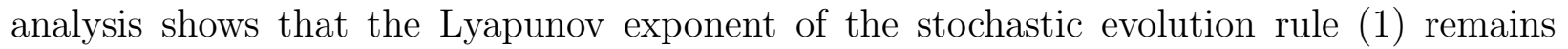
negative for any value of $\sigma$ and, according to Pikovsky's criterion, a synchronized state is always achieved, as we have numerically checked.

In summary, we have presented in this section three different examples of low-dimensional dynamical systems epitomizing possible scenarios concerning the phenomenon of stochastic synchronization: In the last example it occurs for any value of the noise amplitude $\sigma$, in the second one for no value of $\sigma$ and only above some threshold value, $\sigma_{\Lambda}$, in the first example.

\section{STOCHASTIC SYNCHRONIZATION IN CML MODELS}

The generalization of the stochastic map (1]) to a CML model [7] with additive spatiotemporal noise can be defined by the following two-step evolution rule:

$$
\begin{aligned}
& \tilde{x}_{i}^{t}=(1-\varepsilon) x_{i}^{t}+\frac{\varepsilon}{2}\left(x_{i-1}^{t}+x_{i+1}^{t}\right) \\
& x_{i}^{t+1}=f\left(\tilde{x}_{i}^{t}\right)+\sigma \cdot \eta_{i}^{t}
\end{aligned}
$$

The real state variable $x_{i}^{t}$ depends now also on the discrete space index $i=1,2, \cdots, L$ : assuming unit lattice spacing, $L$ corresponds to the lattice size. The strength of the spatial coupling between nearest neighbour maps in the lattice is fixed by the parameter $\varepsilon$, that can take values in the interval $[0,1]$. Note that this kind of coupling amounts to a discrete version of a diffusive term. The application of the map $f$ mimicks the reaction term of reactiondiffusion PDE's, so that CMLs are commonly assumed to represent a sort of discretized version of continuous PDE's. At variance with standard CML models, dynamics (8) contains also a stochastic term given by a set of identical, independent, equally distributed (i.i.e.d.) random variables $\left\{\eta_{i}\right\}$, whose amplitude is determined by the parameter $\sigma$. In what follows these i.i.e.d. random variables are assumed to be uniformly distributed in the interval $[-1,1]$.

In full analogy with the low-dimensional case discussed in the previous section, stochastic synchronization can be investigated by considering two different initial conditions $\left\{x_{i}^{0}\right\}$ and $\left\{y_{i}^{0}\right\}$ for dynamics (8), coupled by the same realization of additive spatio-temporal noise. More precisely, we assume that the corresponding trajectories, $\left\{x_{i}^{t}\right\}$ and $\left\{y_{i}^{t}\right\}$, generated by (8) may synchronize if their distance

$$
d(t)=\frac{1}{N} \sum_{i=1}^{N}\left|x_{i}^{t}-y_{i}^{t}\right|
$$

becomes smaller than a given threshold $\Delta$, usually assumed much smaller than unit. Upon this definition one can straightforwardly extend to the CML case indicators like the first passage time $\tau_{1}(\Delta)$ and the synchronization time $\tau_{2}(\Delta)$. Also in this case we assume that the trajectories synchronize if after the time $\tau_{1}(\Delta), \tau_{2}(\Delta)$ is found to diverge with the integration time. In all the numerical simulations we have used the same value of $\Delta$ as in the low-dimensional case. In fact, we have verified that also for the high-dimensional case 
the results of numerical simulations are not affected by the choice of $\Delta$, provided it is small enough, tipically $\Delta<10^{-7}$. Again, both $\tau_{1}(\Delta)$ and $\tau_{2}(\Delta)$ are quantities averaged over initial conditions and over realizations of noise.

In analogy with low-dimensional systems, we expect that the suitable dynamical indicator for identifying stochastic synchronization in dynamics (8) is the maximum Lyapunov exponent, $\Lambda$. In particular the phenomenon is expected to occur only for values of the noise amplitude $\sigma$ such that $\Lambda$ is negative. For what concerns the interpretation of this indicator for the stochastic CML dynamics (8), the same kind of remarks and conclusions discussed in Section I for the low dimensional case still hold. All the numerical estimates of $\Lambda$ have been performed by applying the standard algorithm outlined in [8].

Another relevant indicator, strictly related to the spatial structure of CML dynamics, is the average propagation velocity of finite amplitude perturbations [9]

$$
V=\lim _{t \rightarrow \infty} \lim _{L \rightarrow \infty} \frac{\langle N(t)\rangle}{2 t}
$$

where

$$
N(t)=\sum_{i=1}^{L} h_{i}^{t} \quad \text { with } \quad h_{i}^{t}= \begin{cases}1 & \text { if }\left|x_{i}^{t}-y_{i}^{t}\right|>0 \\ 0 & \text { otherwise }\end{cases}
$$

is the number of non-synchronized or "infected" sites at time $t$. Here $\left\{x_{i}^{t}\right\}$ and $\left\{y_{i}^{t}\right\}$ represent the trajectories generated by dynamics (8) starting from two initial conditions that differ of finite amounts $\delta_{i} \sim \mathcal{O}(1)$ only inside a space region of size $S$ :

$$
y_{i}^{0}= \begin{cases}x_{i}^{0}+\delta_{i} & \text { if }|L / 2-i| \leq S / 2 \\ x_{i}^{0} & \text { otherwise }\end{cases}
$$

The average $<\cdot>$ in $(10)$ is performed over different initial conditions and noise realizations. The indicator $V$ measures the rate of information propagation : we want to point out that $V$ can take finite values even for non-chaotic evolution, i.e. for $\Lambda<0$. For instance, this scenario has been observed for CMLs made of discontinuous maps [13]. In this case infinitesimal perturbations are unable to be amplified, while finite amplitude perturbations, induced by the discontinuity, can propagate thanks to the spatial coupling. Therefore in spatially extended systems the information flow is absent only when $V$ vanishes.

As a first example let us consider the stochastic CML dynamics (8), equipped with map (5) for $b=2.0$ (see Fig.(1). In this case, numerical simulations indicate that the stochastic synchronization of trajectories occurs for any value of the diffusive coupling $\varepsilon$ and for sufficiently large values of $\sigma$, above which $\Lambda$ becomes negative. For instance, with $\varepsilon=1 / 3$ we find that synchronization is obtained for $\sigma>\sigma_{\Lambda}=2.4768$. This exemplifies the validity of Pikovsky's criterion also for spatially extended systems.

Let us provide more details of the dynamics below and above the threshold value $\sigma_{\Lambda}$. For $\sigma<\sigma_{\Lambda}$ we find that $\tau_{1}(\Delta)$ diverges exponentially with the system's size $L$, i.e. $\tau_{1} \sim$ $\exp (L / \xi)$. The length scale factor $\xi$ is found to be independent of $L$ and proportional to the inverse decay rate of the space correlation function of $x_{i}^{t}$. Accordingly, $L / \xi$ is an estimate of the number of effective independent degrees of freedom. In this dynamical regime the probability $P(\xi, \Delta)$ that two trajectories get closer than a distance $\Delta$ is proportional to 
the combined probability that each one of the $L / \xi$ degrees of freedom get closer than $\Delta$, i.e. $P(\xi, \Delta) \propto \Delta^{L / \xi}$. One can reasonably assume $\tau_{1}^{-1} \propto P(\xi, \Delta)$; this rough argument explains why $\tau_{1} \sim \exp (L / \xi)$. Note that even if $d(t)$ eventually becomes smaller than $\Delta$ synchronization is rapidly lost because of the linear instability mechanism due to positive $\Lambda$ and $\tau_{2}$ is always finite.

Conversely, a logarithmic dependence of $\tau_{1}(\Delta)$ on $L$ is found for $\sigma>\sigma_{\Lambda}$ (see Fig. 2 (b)). Numerical simulations show that in this case the number of regions made of a few synchronized sites increases as time flows. Moreover, once a synchronized region is formed, it grows linearly in time, until all regions merge and synchronization sets in over the whole lattice. One can introduce an argument accounting for the logarithmic dependence $\tau_{1} \propto \ln (L)$. An effective rate equation for the number of synchronized sites, $n(t)$, can be constructed by assigning a probability $p$ for the formation of new synchronized sites and a rate $\gamma$ for the linear increase of synchronized regions:

$$
\frac{\partial n}{\partial t}=\gamma+p(L-n)
$$

with $0 \leq n(t) \leq L$. This equation can be solved with the initial condition $n(0)=0$, so that an estimate of $\tau_{1}$ is obtained by imposing the condition $n\left(\tau_{1}\right)=L$ :

$$
\tau_{1}=\frac{1}{p} \ln \left[\frac{p L}{\gamma}+1\right] .
$$

Note that a logarithmic dependence on $L$ is consistent with the condition $\frac{p L}{\gamma}>>1$, that can be satisfied for sufficiently large values of $L$. By estimating the parameters $p$ and $\gamma$ directly from numerics we have verified that they fit reasonably well the simple phenomenological eq. (14).

The indicator $V$ does not provide any additional information about this kind of synchronization transition. Actually, $V$ is found to be positive for $\sigma<\sigma_{\Lambda}$, while it vanishes at $\sigma=\sigma_{\Lambda}$. This implies that in this model the linear mechanism of information production associated with a positive $\Lambda$ rules also the propagation of finite amplitude perturbations [17]. In summary, for $\sigma>\sigma_{\Lambda} \Lambda$ is negative and $V=0$, so that after the trajectories have reached $\tau_{1}$ it seems that no information production mechanism can be responsible of the resurgence of $d(t)$ above the threshold $\Delta$. This is confirmed by numerical simulations, although in principle one could not exclude that the occurrence of a temporarily positive exponential expansion rate at some lattice site might produce a local amplification of $d(t)$. In this respect, we want to remark that $\Lambda$ is a global indicator, i.e. the exponential expansion rate between nearby orbits averaged in time and over the whole phase space. Accordingly a negative value of $\Lambda$ is fully compatible with the above mentioned local, instantaneous event. This point, that reveals important for the understanding of the dynamical mechanisms underlying the synchronization transition, will be analyzed more carefully in the following section.

Different scenarios are obtained by considering dynamics (8) with $f$ given by the logistic map (6). For $\varepsilon=1 / 3$ and large enough values of $L$, one recovers features very similar to the case of a single logistic map, where the synchronization transition is absent. In fact, for any value of $\sigma, \Lambda$ and $V$ are positive, while $\tau_{1}$ and $\tau_{2}$ remain finite. Moreover $\tau_{1}$ is found to diverge exponentially with $L$ with a parameter dependent rate. 
A different situation occurs for $\varepsilon=2 / 3$, where $\Lambda$ vanishes at $\sigma_{\Lambda}=0.27$, while $V$ remains positive up to $\sigma_{V} \approx 0.4$ (see Fig. 3). According to Pikovsky's criterion one expects that synchronization occurs above $\sigma_{\Lambda}$. This is indeed the case, although, for $\sigma_{\Lambda}<\sigma<\sigma_{V}, \tau_{1}(\Delta)$ is still found to increase exponentially with $L$, while for $\sigma>\sigma_{V}, \tau_{1}(\Delta)$ grows logarithmically with $L$ (see Fig. 1). Despite the strong analogy between this transition at $\sigma_{V}$ and the one occurring in the first example at $\sigma_{\Lambda}$, we want to remark that there is a crucial difference between them: below threshold $\tau_{2}$ diverges in the former case, while it is finite in the latter.

This indicates the existence of a new kind of synchronization transition at $\sigma_{V}$ essentially ruled by $V$. Let us point out that, at variance with the first example, for $\sigma_{\Lambda}<\sigma<\sigma_{V}$ the non-linear mechanism of information propagation is enough for maintaining the exponential dependence of $\tau_{1}$ on $L$. On the other hand, after $\tau_{1}$ two trajectories get very close to each other and the negative $\Lambda$ stabilizes them onto the same stochastic trajectory.

Let us stress that the above described scenarios are not peculiar of the specific choice of the parameter values $\varepsilon=1 / 3$ and $\varepsilon=2 / 3$. We have checked that their main features are robust w.r.t. small but finite variations of $\varepsilon$, although a systematic investigation of the parameter space would demand too huge computational times.

Upon these examples one can conclude that in a CML of very large but finite size $L$ stochastic synchronization of two trajectories within an accessible time span occurs when $V$ vanishes.

Moreover, a very similar situation is obtained when considering dynamics (8) for the model of period-3 stable maps (7) introduced in [13. In this CML model $\Lambda$ is found to be negative, independently of the value of the diffusive coupling parameter $\varepsilon$ and, accordinlgy, the dynamics eventually approaches a periodic attractor. On the other hand, the model exhibits a transition between a frozen disordered phase with $V=0$ to a chaotic phase with $V>0$ at $\varepsilon_{c} \approx 0.6$. The peculiar feature of this transition is that these two phases are separated by a small fuzzy region centered around $\varepsilon_{c}$, where both positive and null values of $V$ can be observed up to available numerical resolution.

The addition of noise to this CML dynamics according to eq. (8) has interesting consequences: $\Lambda$ is kept negative independently of the noise amplitude $\sigma$, while a small amplitude noise destabilizes the frozen disordered phase: $V$ becomes positive even for $\varepsilon<\varepsilon_{c}$, so that the fuzzy transition disappears. This notwithstanding, by increasing $\sigma$ up to a critical value $\sigma_{V}(\varepsilon), V$ is found to drop again to zero not only below, but also above $\varepsilon_{c}$. For instance, one has $\sigma_{V} \approx 0.16$ for $\varepsilon=0.58$ and $\sigma_{V} \approx 0.18$ for $\varepsilon=0.62$. In both cases we recover the same kind of mechanisms characterizing the synchronization transition discussed for coupled logistic maps with $\varepsilon=2 / 3$.

We want to point out that a finite value of $V$, when $\Lambda$ is negative, is usually reported as a typical signature of a strong non-linear effect [17]. For instance, it has been shown 13] that the discontinuity of map (7) yields such an effect already for the noise-free CML dynamics. Even if the discontinuity of map (7) is removed by interpolating the expanding and contracting regions with a sufficiently steep segment the effect is maintained [19].

The reinjection mechanism introduced by additive noise in maps of the interval $[0,1]$ into itself produces similar discontinuities in the dynamics. As we have shown this non-linear effect is sufficient for giving raise to dynamical phases with $\Lambda<0$ and $V>0$ in the noisy dynamics (8).

All these observations suggest to investigate if a similar scenario can be obtained for map 
(5) in dynamics (8), by introducing an almost-discontinuity in the map, since in this case the reinjection mechanism is not present. This is easily obtained by taking a sufficiently large value of the parameter $b$, e.g. $b \sim \mathcal{O}\left(10^{3}\right)$. Numerical simuations show that, still for $\varepsilon=1 / 3$, it exists a range of $\sigma$-values for which $V>0$ and $\Lambda<0$. In Fig.5 we show the dependence of $\tau_{1}$ on $L$ in two regions of the parameter space where $\Lambda$ is negative, while $V$ is either positive or null. If $V>0$ an exponential increases of $\tau_{1}$ with $L$ is again observed, while a logarithmic dependence characterizes the dynamical phase with $V=0$.

Accordingly, this second kind of transition is not just an artifact due to discontinuities introduced by the rejection rule, but a mere consequence of sufficiently strong non-linear effects, that may be produced also in the absence of any rejection mechanism and also if the noise distribution maintains its symmetry.

\section{CRITICAL PROPERTIES OF THE SYNCHRONIZATION TRANSITION IN CMLS}

In the previous Section we have described two different kinds of phase transitions for stochastic synchronization where the noise amplitude $\sigma$ plays the role of the control parameter. When both $\Lambda$ and $V$ vanish at $\sigma=\sigma_{\Lambda}$ the transition is from a non-synchronous dynamical phase to a synchronous one: we have denoted it with PT1. In the other case, that we indicate with PT2, $\Lambda$ passes from positive to negative values while $V$ remains positive up to $\sigma=\sigma_{V}$, where we have observed a transition between different synchronous dynamical phases, characterized by an exponential and by a logarithmic dependence on the system size of the first passage time $\tau_{1}$.

In both cases we have found that the correlation length $\xi$, defined in Sect. II, diverges at the transition point : this is an indication in favour of a continuous phase transition. Nonetheless, $1 / \xi$ is found to vanish less than linearly when $\sigma \rightarrow \sigma_{\Lambda}^{-}$in PT1, while it vanishes linearly in PT2 when $\sigma \rightarrow \sigma_{V}^{-}$(see Figs. 6 (a),(b)).

Moreover, close to the critical point the time averages of the mean distance between trajectories, $z^{t}=\frac{1}{L} \sum_{i=1}^{L}\left|x_{i}^{t}-y_{i}^{t}\right|$, and of the topological distance, $\rho(t) \equiv \frac{1}{L} \sum_{i=1}^{L} h_{i}^{t}\left(h_{i}^{t}=1\right.$ if $\left|z_{i}^{t}\right|>\Delta$, otherwise $h_{1}^{t}=0$ ) exhibits a continuous dependence on $\sigma$ (for the sake of space in Fig.7 we show these quantities only for the case of coupled logistic maps).

We want to remark that our definition of the synchronized state implies that it exists a stable stochastic orbit that prevents the trajectories of the dynamical system to flow apart from each other below some very small, but finite threshold $\Delta$ so that $\tau_{2}=\infty$. In both cases numerical simulations show that this is what happens above the critical point, where $\Lambda<0$ and $V=0$. If at some lattice site $i$ a fluctuation makes the local Lyapunov multiplier positive (i.e., $\ln \left|f^{\prime}\left(\tilde{x}_{i}^{t}\right)\right|>0$, where $f^{\prime}$ indicates the first derivative of the map w.r.t. its argument), giving rise locally to the exponential divergence of nearby orbits, the process is rapidly reabsorbed due to the lack of any mechanism of information propagation. In this sense the synchronized state should be equivalent to the absorbing state, typical of Directed Percolation (DP) processes [11, 12].

Below the critical points the role of fluctuations determines the difference between PT1 and PT2. The inspection of the space-time evolution of dynamics (8), using the symbolic representation of the state variables $h_{i}^{t}$, is quite helpful for visualizing such a difference. For what concerns PT1, when $\sigma \rightarrow \sigma_{\Lambda}^{-}$one observes that non-synchronized clusters propagate 
as time flows (since $V$ is positive) and, even if some of them may eventually die, in the meanwhile new ones have started to propagate, emerging also from previously synchronized regions. Since also $\Lambda>0$ any local fluctuation of $d(t)$ produced by a positive multiplier has a finite probability to be amplified and eventually propagated through the lattice.

On the contrary, sufficiently close to PT2, for $\sigma \rightarrow \sigma_{V}^{-}$, non-synchronized clusters never emerge from already synchronized regions and any connected non-synchronous cluster eventually dies at $\tau_{1}(L, \sigma)$ in a lattice of finite size $L$ (a situation very similar to what is observed in the active phase of DP as a finite size effect). Even if, in principle, the non-linear mechanism of information propagation is active, this suggests that, inside an already synchronized region, any local fluctuation of the Lyapunov multiplier towards positive values never persits long enough to activate the non-linear process of information propagation.

Upon these numerical observations and exploiting the analogies with other critical phenomena, we are led to conjecture not only the existence of an absorbing, i.e. synchronized, state but also that PT2, at variance with PT1, should belong to the universality class of DP. This can be confirmed only by direct measurements of the critical exponents associated with the synchronization transitions. It is worth stressing that numerical estimates have been performed by approaching the critical points from below, i.e. for $V \rightarrow 0^{+}$.

Here we report the analysis of PT2 in the case of coupled logistic maps with $\varepsilon=2 / 3$. As usual a reliable measurement of any critical exponent demands a very accurate estimate of the critical point, i.e. $\sigma_{V}$, in this case. For this reason we have performed careful simulations for evaluating the dependence on the system size $L$ of $\tau_{1}$, that corresponds to the absorption time in the DP language. At the critical point $\sigma=\sigma_{V}$, this time should diverge as

$$
\tau_{1}\left(L, \sigma_{V}\right) \sim L^{z}
$$

where $z=\nu_{\|} / \nu_{\perp}$ is the dynamical exponent [11,12]. The quantity $\tau_{1}(L, \sigma)$ is reported in Fig. 8 as a function of $L$ in a $\log$-log scale for different values of $\sigma$. The best scaling behaviour is obtained for $\sigma_{V}=0.4018$, where one has $z=1.55 \pm 0.05$. This result agrees quite well with the most accurate numerical estimates of the DP value $z=1.5807$ [20]. Relying upon this result, we have also measured the critical exponent associated with the temporal decay of the density $\rho(t)$ of active sites, i.e. those sites where $\left|x_{i}^{t}-y_{i}^{t}\right|>\Delta$. The DP transition exhibits at the critical point the following scaling law for the topological distance: $\rho(t) \sim t^{-\delta}$, with $\delta=\beta / \nu_{\|}=0.1595$ [20]. The density $\rho=\rho(t)$ is shown in Fig. 9 for three different system sizes, namely $L=500,1500$ and 2000 . As expected the scaling region increases with $L$ and for $L=2000$ an optimal fitting in the interval $3.2 \leq \log (t) \leq 4.8$ provides the estimate for the critical exponent $\delta=0.159 \pm 0.002$. This confirms that PT2 belongs to the universality class of DP.

For what concerns PT1 we have considered coupled maps of the type (5) for $b=2.0$ and $\varepsilon=1 / 3$. The best scaling for the $\tau_{1}$ as a function of $L$ (accordingly to equation (15)) is observed for a noise amplitude $\sigma_{\Lambda}=2.5015$. For $\sigma=\sigma_{\Lambda}$ we have obtained the following estimates for the critical exponents : $z \approx 1.01-1.04$ and $\delta \approx 0.35$. Such values certainly do not correspond either to DP, or to any known universality class for percolation or growth processes. This seems analogous to what has been pointed out by Grassberger [12] for systems exhibiting incomplete deaths: when the asymptotic state is not a truly absorbing one the critical properties of DP cannot be recovered.

Since in this situation an absorbing state seems not to exist, the dynamical exponent 
$z$ can be better estimated by measuring the number of "infected" sites of the chain $N(t)$ defined in (11). At the critical point $\sigma=\sigma_{\Lambda}$ the following scaling law is expected to hold

$$
N(t) \sim t^{1 / z}
$$

The data from numerical analysis are reported in Fig. 10. For short times $(t<500)$ we obtain the inverse of the the dynamical exponent $1 / z \sim 0.47 \pm 0.05$, a value consistent with the one expected for the Edwards-Wilkinson universality class $z_{E W}=2.0$ [21]. For longer times we observe a crossover to a lower $z$-value that is consistent with the one expected for the $1 \mathrm{~d}$ KPZ universality class (namely $z_{K P Z}=3 / 2$ ). We think that these results could be interpreted in terms of the conjecture reported in [23]. In that paper it has been suggested that, for generic synchronization transition of coupled spatio-temporal chaotic systems with continuous state variables, the appropriate universality class should be the one of the KPZ model with a non-linear growth limiting term [12,22]. This idea originates from the observation that, close to the synchronization transition, it is possible to describe the dynamics of small perturbations in terms of a reaction diffusion model with multiplicative noise [12]. Finally, this model can be mapped via a Hopf-Cole transformation into an equation that corresponds to a KPZ model with a non-linear term that prevents the surface from growing indefinitely [24]. The critical scaling laws for this kind of models have been reported in [22]: the dynamical exponent $z$ is found to coincide with the KPZ-one, while the other exponents are found to be different from the standard KPZ ones. Unfortunately a full numerical comparison of PT2 with the model discussed in [22] is prevented by two major technical problems: the extreme difficulty to estimate with sufficient precision the critical value $\sigma_{\Lambda}$ and the strong finite size effects.

\section{CONCLUDING REMARKS}

We have studied the problem of stochastic synchronization induced by additive spatiotemporal noise in CML models. In analogy with the low-dimensional case, synchronization of trajectories is observed if the maximum Lyapunov exponent becomes negative at a critical value of the noise amplitude. We have also identified two different critical behaviours associated with the synchronization transition. One of them belongs to the universality class of Directed Percolation. While the other one is not clearly identified, but indications suggest that it could belong to the universality class of the KPZ model with a non-linear growth limiting term.

In particular, the DP-like phase transition describes the crossover between an "active" and an "absorbing" phase. The former is characterized by an exponential dependence on the system size $L$ of the time needed for achieving the synchronized state, while the latter exhibits a logarithmic dependence. This scenario is reminescent of the phenomenology associated with stable-chaos in CMLs [13], where the dynamics approaches a periodic attractor, rather than a "synchronized" stochastic trajectory.

The two kinds of synchronization transition here reported are quite general for extended dynamical systems, since analogous behaviours have been recently observed for two coupled CMLs without any external noise [25].

Moreover, as far as the control of chaos is concerned when the erratic behaviours present in the extended system are due solely to non-linear mechanisms (as it happens when the 
maximal Lyapunov is negative, but $V$ is still positive) the control schemes based on linear analysis [26] should fail and new "non-linear" methods have to be introduced. We believe that the appropriate indicator to employ in this context is the Finite Size Lyapunov Exponent [27], because it is able to capture infinitesimal as well as finite amplitude perturbation growth.

We also expect that the observed phenomenology is not peculiar of CMLs and the present study can apply to a wider class of spatially extended dynamical systems, like coupled oscillator or PDE's.

\section{ACKNOWLEDGMENTS}

We want to thank all the components of the D.O.C.S research group of Firenze for stimulating discussions and interactions, and in particular F. Bagnoli and A. Politi. We express our gratitude also to V. Ahlers, M.A. Muñoz, A. Pikovsky and P. Grassberger for helpful discussions (P.G. also for providing us the efficient random number generator that we have employed in numerical simulations). A.T. acknoweledges the contribution of T. Caterina, H. Katharina, H. Daniel amd T. Sara for providing him a realistic realization of a chaotic and noisy enviroment. Part of this work was performed at the Institute of Scientific Interchange in Torino, during the workshop on "Complexity and Chaos", June 1998 and October 1999. We acknowledge CINECA in Bologna and INFM for providing us access to the parallel computer CRAY T3E under the grant "Iniziativa Calcolo Parallelo". 


\section{REFERENCES}

[1] L.M. Pecora and T.L. Carroll, Phys. Rev. Lett. 64821 (1990).

[2] A. S. Pikovsky, Phys. Lett. A 165, 33 (1992).

[3] S. Fahy and D.R. Hamann, Phys. Rev. Lett. 69, 761 (1992); A. Maritan and J. R. Banavar, Phys. Rev. Lett. 72, 1451 (1994) and 73, 2932 (1994).

[4] A. S. Pikovsky, Phys. Rev. Lett. 73, 2931 (1994).

[5] H. Herzel and J. Freund, Phys. Rev. E 52, 3238 (1995).

[6] C.H. Lai and Changsong Zhou, Europhys. Lett. 43, 376 (1998).

[7] K. Kaneko, Prog. Theor. Phys. 72, 980 (1984); I. Waller and R. Kapral, Phys. Rev. A 30,2047 (1984).

[8] I. Shimada and T. Nagashima, Prog. Theor. Phys. 61, 1605 (1979); G. Benettin, L. Galgani, A. Giorgilli and J.M. Strelcyn, Meccanica, March 15 and 21 (1980).

[9] P. Grassberger, Physica Scripta, 40, 1033 (1985); K. Kaneko, Physica D, 23, 436 (1986).

[10] M. Kardar, G. Parisi and Y.-C. Zhang, Phys. Rev. Lett. 56, 889 (1986).

[11] E. Domany and W. Kinzel, Phys. Rev. Lett. 53, 311 (1984).

[12] For a recent review on Directed Percolation see P. Grassberger, Directed Percolation: Results and Open Problems, in Nonlinearities in Complex Systems, Prooceedings of 1995 Shimla Conference on Complex Systems, eds. S. Puri et al. (Narosa Publishing, New Dehli, 1997).

[13] A. Politi, R. Livi, R. Oppo, and R. Kapral, Europhys. Lett. 22, 571 (1993); R. Kapral, R. Livi, R. Oppo, and A. Politi, Phys. Rev. E 49, 2009 (1194).

[14] L. Baroni, R. Livi, and A. Torcini, Noise-driven Synchronization in Coupled Map Lattices, published in "Dynamical Systems:from Cristal to Chaos", eds J-M Gambaudo, P Hubert, P Tisseur, and S Vaienti (World Scientific, Singapore, 2000 ) page 23.

[15] We have checked that different norms, e.g. the maximum or the euclidean ones, do not modify the scenario described herein. Note that both quantities are well defined also in the noise-free case, since there is a finite probability that two different initial conditions may stay closer than a finite distance $\Delta$.

[16] In a recent book by L. Arnold, Random dynamical systems, in "Springer monographs in mathematics", New York - Berlin 1998, it has been shown that the maximum Lyapunov exponent is a meaningful quantity when the deterministic dynamics is coupled with e time independent stochastic signal.

[17] A. Torcini, P. Grassberger and A. Politi, J. Phys. A : Math. Gen. 28, 4533 (1995).

[18] F. Cecconi, R. Livi and A. Politi, Phys. Rev. E E 57, 2703 (1998).

[19] A. Politi and A. Torcini, Europhys. Lett. 28 (1994) 545.

[20] I. Jensen, J. Phys. A 29, 7013 (1996); Low-density series expansions for directed percolation I: A new efficient algorithm with applications to the square lattice, condmat/9906036 (unpublished).

[21] A.-L. Barabasi and H.E. Stanley Fractal Concepts in Surface Growth (Cambridge University Press, Cambridge, 1995).

[22] G. Grinstein, M.A. Muñoz, and Y. Tu, Phys. Rev. Lett. 76, 4376 (1996); Y. Tu, G. Grinstein, and M.A. Muñoz, Phys. Rev. Lett. 78, 274 (1997).

[23] P. Grassberger, Phys. Rev. E 59, R2520 (1999).

[24] A. S. Pikovsky and J. Kurths, Phys. Rev. E 49, 898 (1994).

[25] V. Ahlers and A. S. Pikovsky, private communication. 
[26] E. Ott, C. Grebogi, and J.A. Yorke, Phys. Rev. Lett. 64, 1196 (1990).

[27] E. Aurell, G. Boffetta, A. Crisanti, G. Paladin, and A. Vulpiani, Phys. Rev. Lett. 77, 1262 (1996); M. Cencini and A. Torcini, A nonlinear marginal stability criterion for information propagation, submitted to Phys. Rev. E in 2000. 


\section{FIGURES}

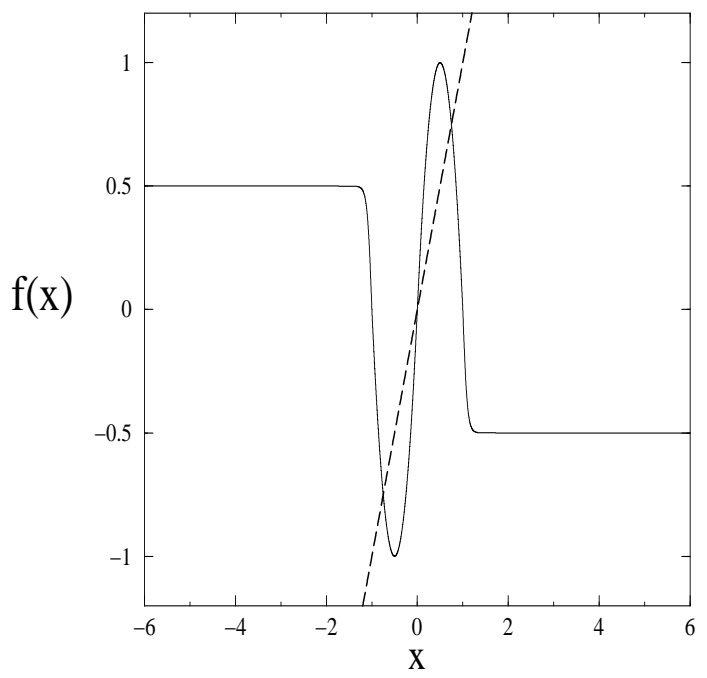

FIG. 1. The map (5) for $a=4, b=2$ and $c=0.5$.
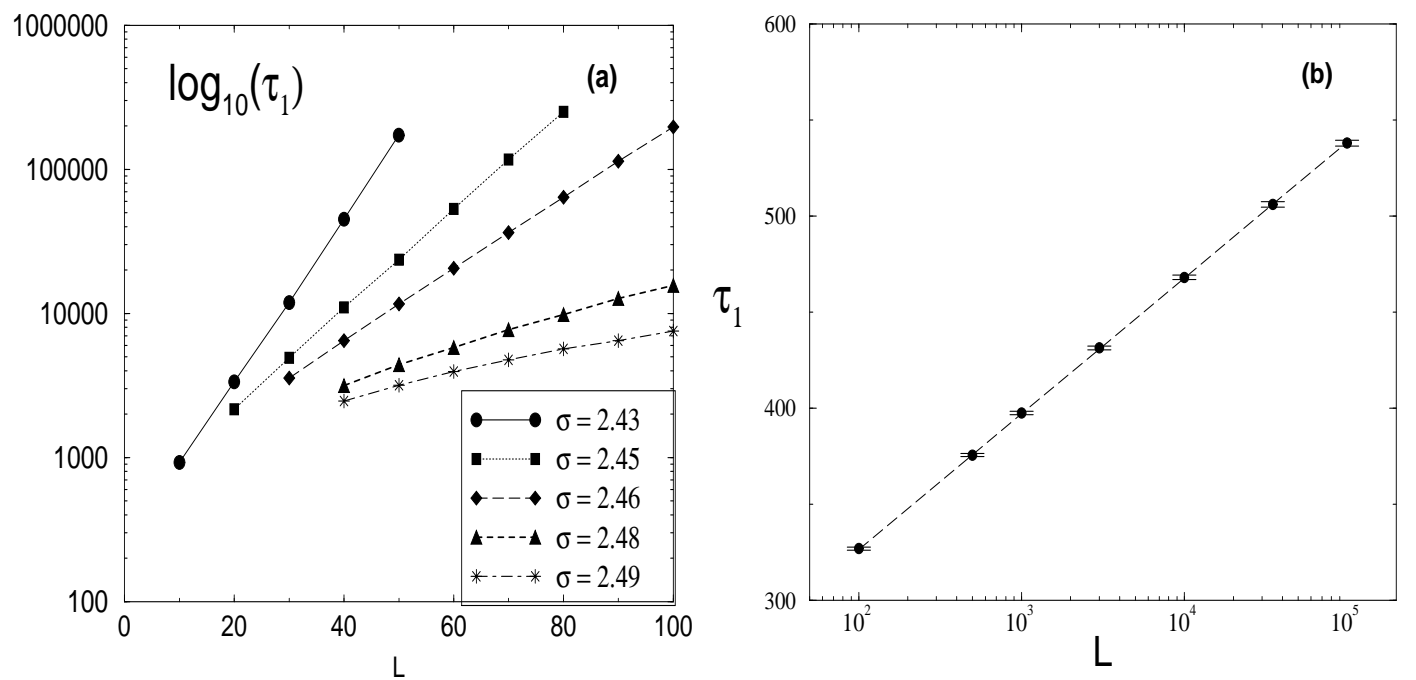

FIG. 2. $\tau_{1}$ versus $L$ for coupled maps (5) for $a=4, b=2, c=0.5$, with $\varepsilon=1 / 3$ : a) exponential scaling observed for $2.49 \leq \sigma \leq 2.43$; b) logarithmic scaling for $\sigma=2.6$ (filled circles). The values of $\tau_{1}$ have been computed with $\Delta=10^{-8}-10^{-12}$ and averaged over $1000-5000$ initial conditions. 


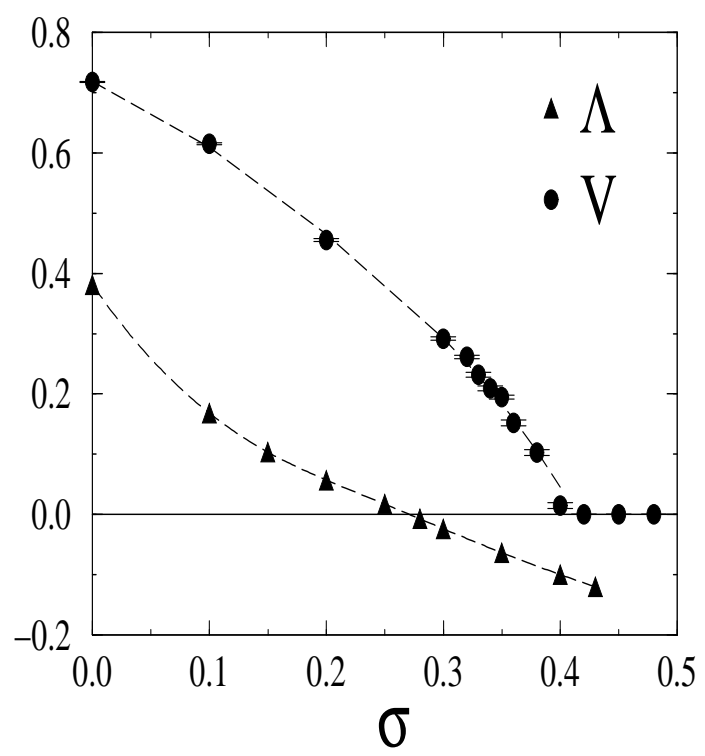

FIG. 3. Behaviour of the propagation velocity $V$ (circles) and of the maximum Lyapunov exponent $\Lambda$ (triangles) versus noise amplitude $\sigma$ for coupled logistic maps with $\varepsilon=2 / 3$. Both quantities have been computed for $L=1024$, averaging over $10^{3}$ initial conditions each one followed for $\mathcal{O}\left(10^{5}\right)$ time steps. The dashed lines are a guide for the eyes.

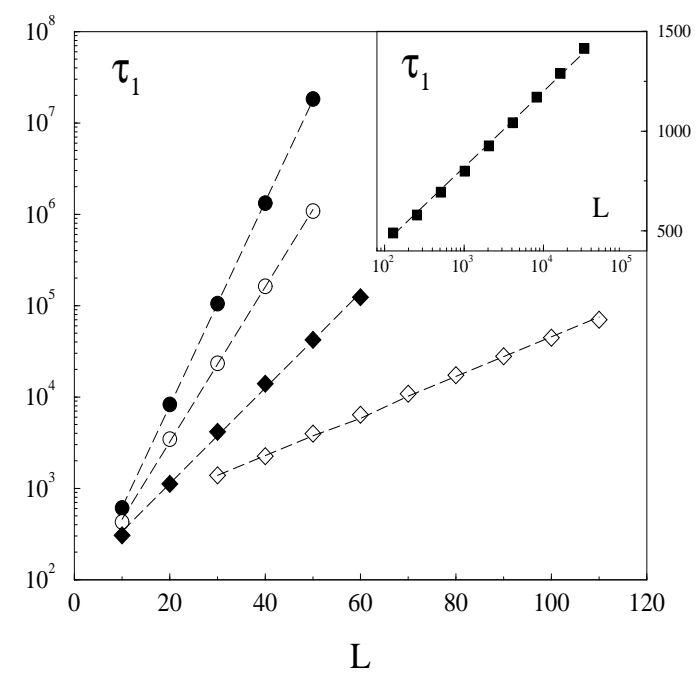

FIG. 4. Exponential scaling of $\tau_{1}$ versus $L$ (reported in a lin-log scale) for coupled logistic maps with $\varepsilon=2 / 3$ at different values of the noise amplitude: $\sigma=0.3$ (filled circles), 0.32 (empty circles), 0.35 (filled diamonds), 0.38 (empty diamonds). The inset shows the logarithmic scaling of $\tau_{1}$ versus $L$ (in log-lin scale) for $\sigma=0.45$. The values of $\tau_{1}$ have been computed with $\Delta=10^{-10}$ and averaged over $10^{3}$ initial conditions. 

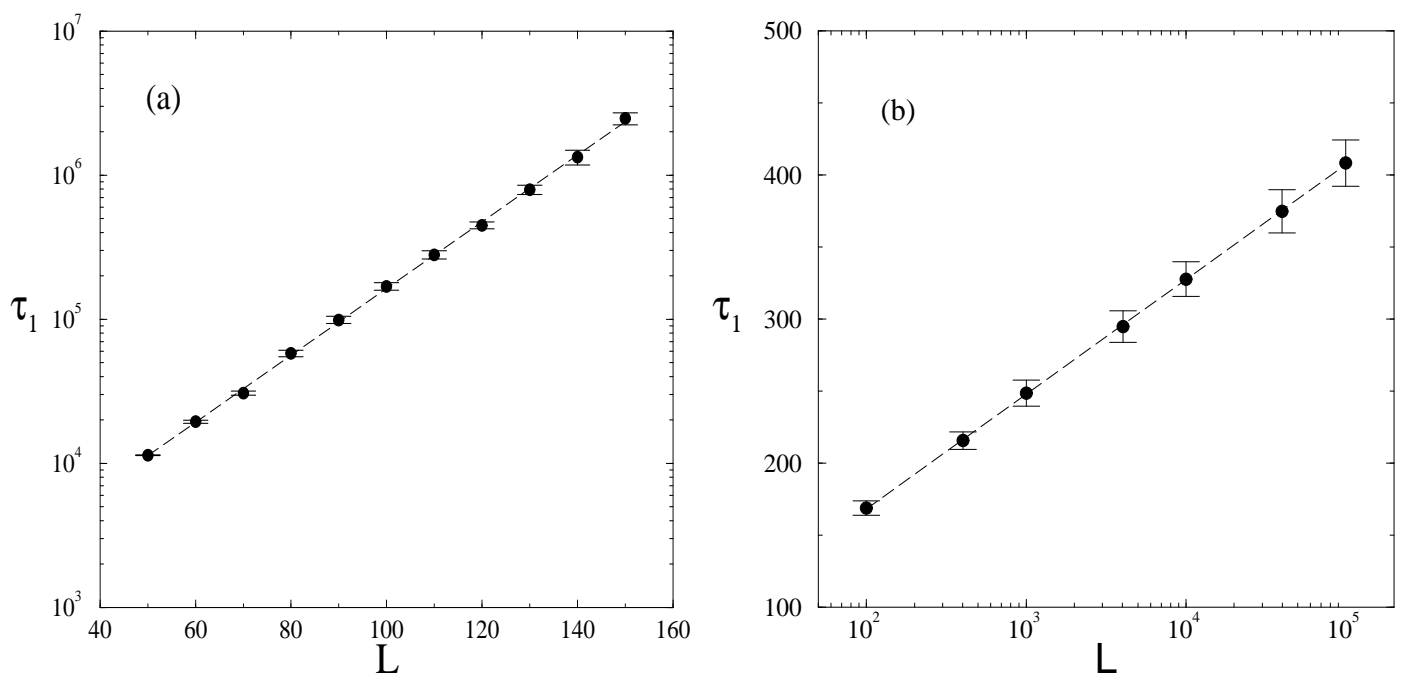

FIG. 5. (a) Exponential scaling of $\tau_{1}$ versus $L$ (reported in lin-log scale) for coupled maps (5) for $a=4, b=1000, c=0.5$, with $\varepsilon=1 / 3$ and $\sigma=2.1$ (filled circles). (b) Logarithmic scaling of $\tau_{1}$ versus $L$ (shown in log-lin scale) for $\sigma=2.5$ (filled circles). In this case $\sigma_{\Lambda}=1.99$ and $\sigma_{V}=2.15$. The values of $\tau_{1}$ have been computed with $\Delta=10^{-8}$ and averaged over $10^{3}$ initial conditions.
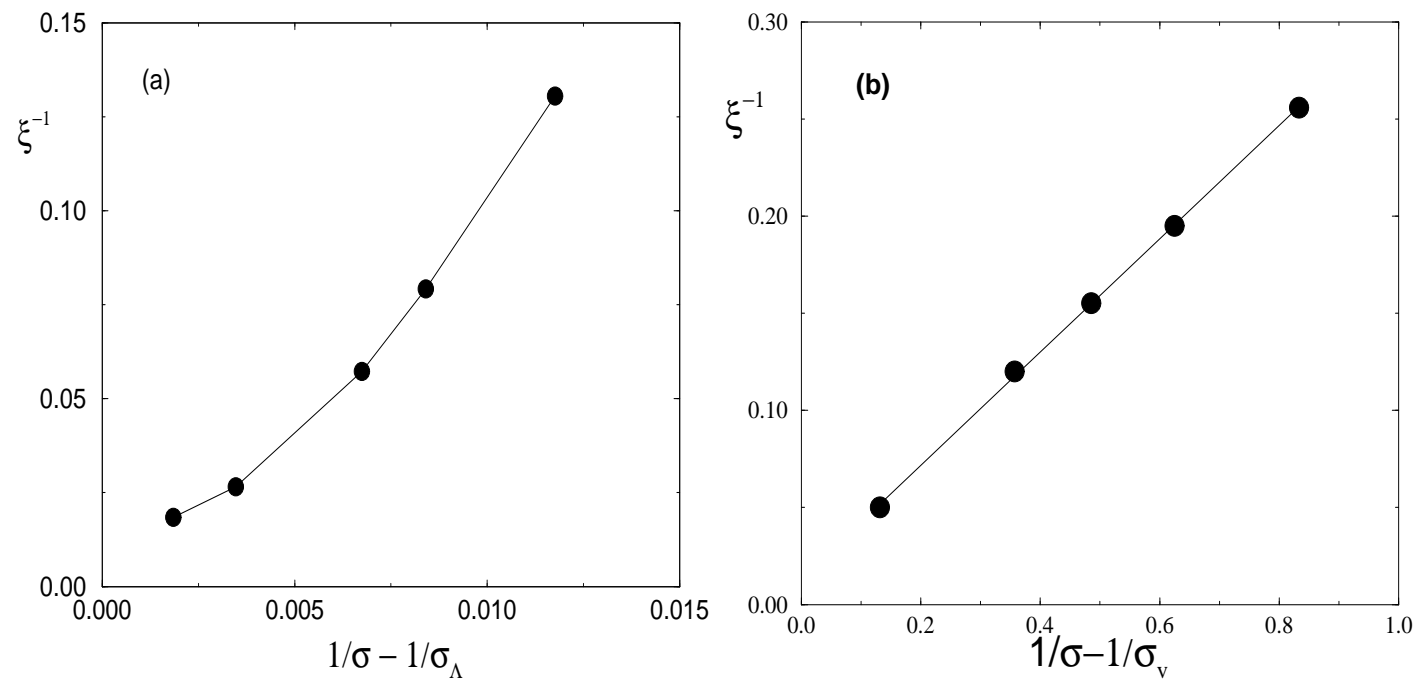

FIG. 6. (a) The inverse of the scaling factor $1 / \xi$ as a function of $\sigma^{-1}-\sigma_{\Lambda}^{-1}\left(\sigma_{\Lambda}=2.5015\right)$ is reported for coupled (5) maps with $b=2.0$ and $\varepsilon=1 / 3$ and $2.49 \leq \sigma \leq 2.43$; (b) $1 / \xi$ as a function of $\sigma^{-1}-\sigma_{V}^{-1}\left(\sigma_{V}=0.4018\right)$ for coupled logistic maps with $\varepsilon=2 / 3$ and $0.30 \leq \sigma \leq 0.38$. 

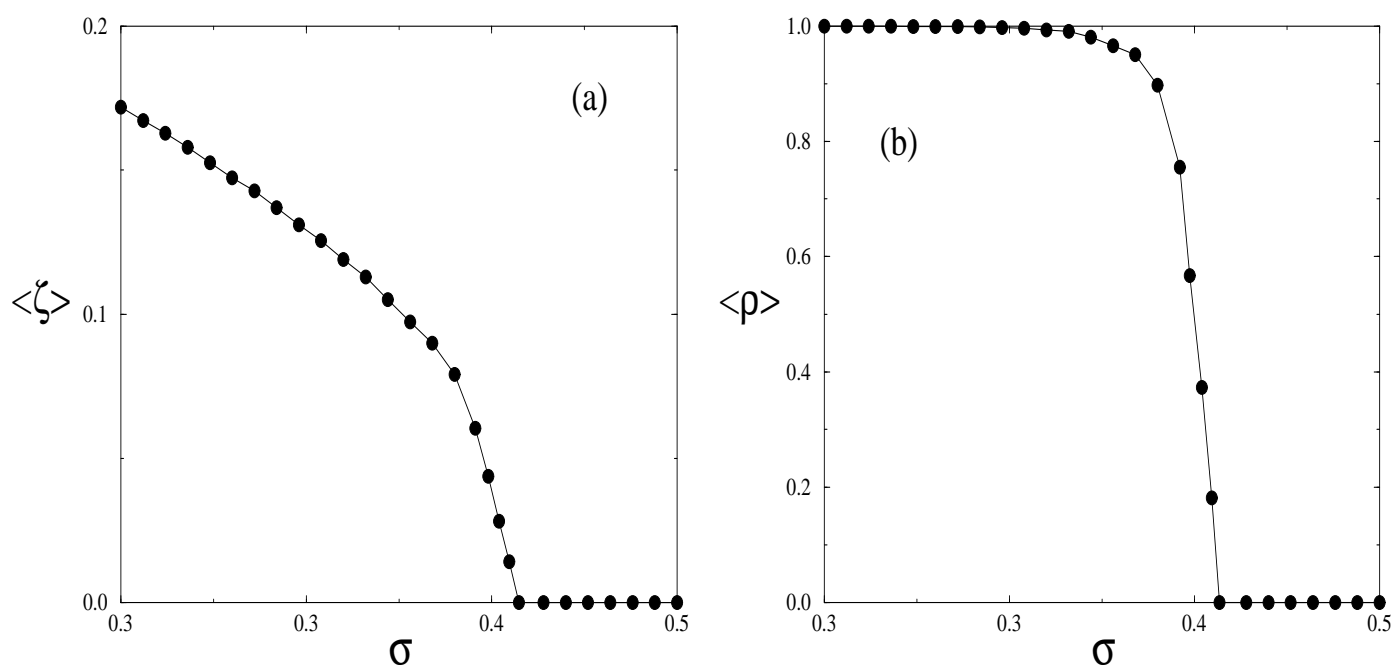

FIG. 7. The spatio-temporal averages of the indicators $\zeta$ (a) and $\rho$ (b) are reported as a function of the noise amplitude $\sigma$ for for coupled logistic maps with $\varepsilon=2 / 3$. The data have been obtained by averaging over a time $t=10^{4}$ and 20 different initial conditions, for $L=4096$ and considering a threshold $\Delta=10^{-12}$.

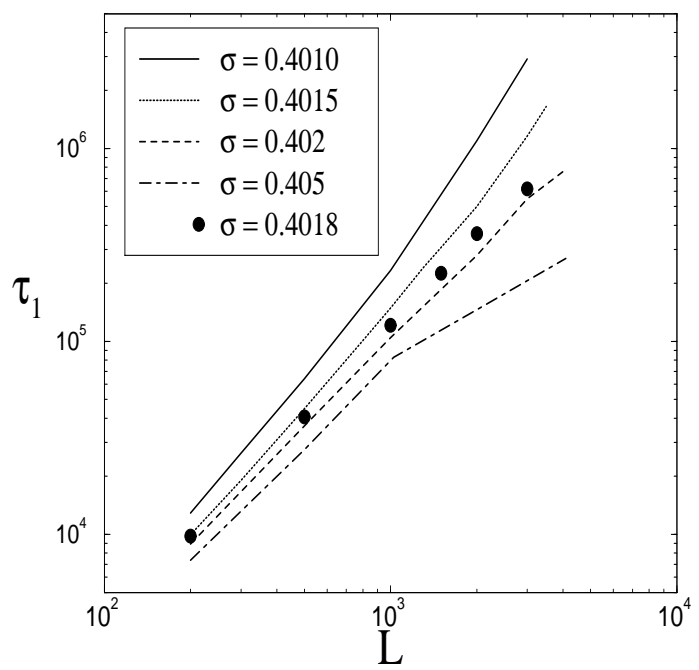

FIG. 8. The first passage time $\tau_{1}$ is reported in a log-log scale as a function of system size $L$ for coupled logistic maps with $\varepsilon=2 / 3$ and for various $\sigma$. The data have been obtained by averaging over 3,000 - 25,000 different initial conditions and considering a threshold $\Delta=10^{-12}$. 


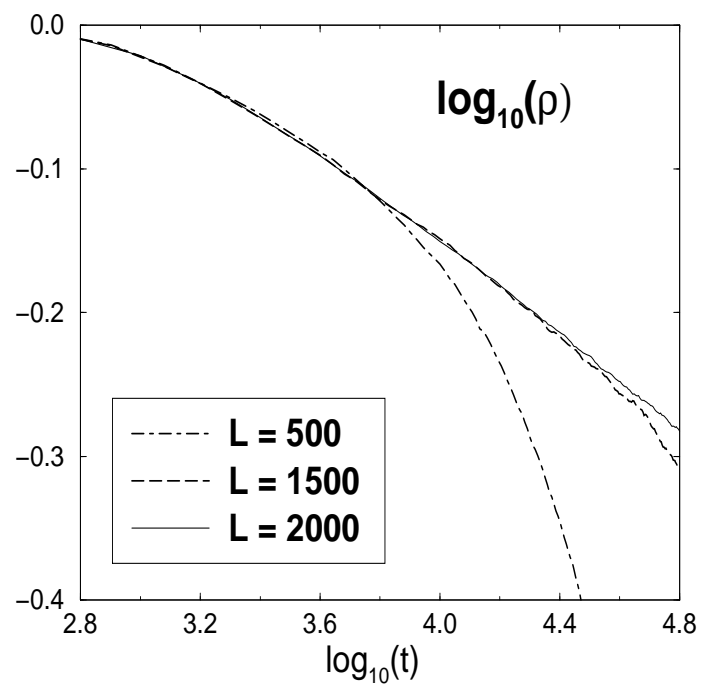

FIG. 9. Logarithm in base 10 of the density of active sites as a function of $\log _{10}(t)$ for coupled logistic maps with $\varepsilon=2 / 3$ and $\sigma=0.4018$. The data have been obtained by averaging over 10,000 different initial conditions and considering a threshold $\Delta=10^{-15}$.

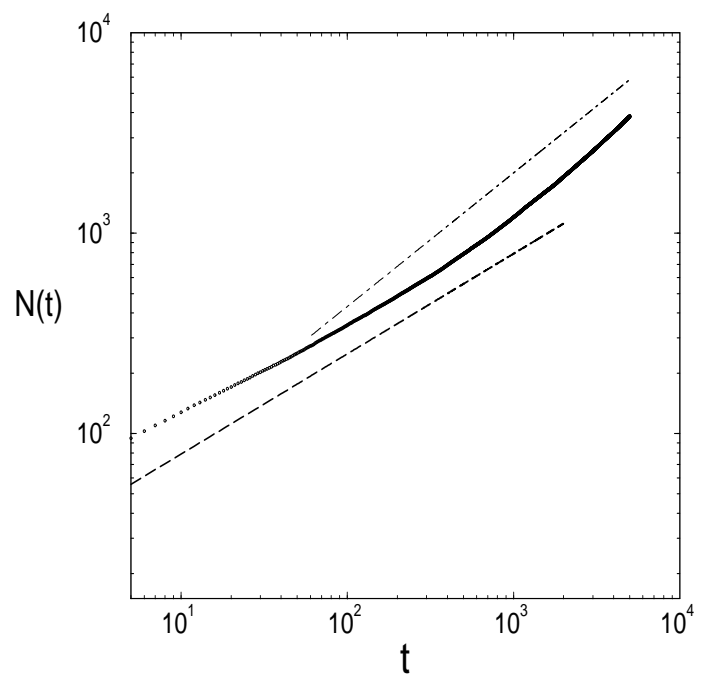

FIG. 10. The number of "infected" sites $N(t)$ is reported in a log-log scale as a function of time $t$ for coupled maps (5) with $a=4, b=2, c=0.5$ and $\varepsilon=1 / 3$ and for various $\sigma$. The numerical data (filled circles) have been obtained for a chain length $L=20,000$ by averaging over 1,000 different initial conditions and considering a threshold $\Delta=10^{-15}$. The dashed line indicates a scaling of the Edwards-Wilkinson type (with exponent $1 / z=0.5$ ) while the dot-dashed line has a slope $1 / z=2 / 3$ analogous to that of the KPZ scaling. 